\title{
Microm Ergostar HM200 Microtome
}

National Cancer Institute

\section{Source}

National Cancer Institute. Microm Ergostar HM200 Microtome. NCI Thesaurus. Code C126389.

A multi-purpose, proprietary specimen preparation system that integrates rotary and sliding microtomes. 